\title{
Comparison between relative wear resistance of flowable and highly filled composite
}

\author{
Ivan Chakalov' ${ }^{1}$, Pavlina Koleva ${ }^{2}$, Ivan \\ Gerzhikov $^{1}$, Nickolay Apostolov ${ }^{1}$
}

1. Department of Prosthetic Dentistry, Faculty of Dental Medicine, Medical University - Sofia, Bulgaria;

2. Dentist with a Private Practice - Sofia, Bulgaria;

\begin{abstract}
Traditional flowable composites are known to adapt well to cavity preparations and are easy to apply. They spare clinical time as placing a flowable into a cavity preparation is a fairly easy task compared to having to pack a thicker composite, especially in areas such as a proximal box. The latest trend regarding flowables is the claim that some of them are wear resistant enough to be used for the entire restoration regardless of the classification. This means these specialized flowables can presumably be used to restore even Class II and IV lesions and/or fractures. G-aenial Universal Flo (GC) is being tagged as the "first injectable flowable" that "can also be used as a restorative". Voco is promoting Grandio Flow as the "first flowable composite that is strong as universal composites" and Shofu is bringing to the market Beautifil Flow Plus, which is stated to be "a flowable for complete anterior and posterior restorations". In the field of esthetic dentistry flowables are sometimes even applied on the occlusal surface of restorations as an artistic refinement and finishing touch. But as every new trend it is worth putting it into question and testing the wear resistance of flowables.
\end{abstract}


We conducted an in-vitro research in order to compare the wear resistance of flowable composite (SDI Wave) and highly filled, packable composite (Herculite XRV, Kerr) using the chewing simulator "Sofia". The results presented lower wear resistance of flowables confirming the outcomes of previous researches (1). Therefore the indication for use of flowable composites have some limitation despite the manufacturers' claims.

Keywords: flowable composite, wear resistance, in-vitro tests, chewing simulator, highly filled composite

\section{Introduction}

Light-cured flowable composites were introduced to the dental profession for the first time in 1995 with Revolution and it was indicated for Class III, IV, and V restorations, porcelain veneer cementation, porcelain and marginal defect repairs, sealants, and core buildups (2). Since then the dental technology has rapidly developed and the clinical performance of dental composites has been significantly improved through modifications in formulation that include: using more stable polymerization promoters for greater color stability; incorporating high concentrations of finely ground fillers to produce adequate strength and excellent wear resistance while retaining translucency; adding radiopacifying agents for improved diagnostics; and utilizing dentin adhesives (3). Nowadays new generations of flowable composites are launched on the market such as highly filled flowable composites indicated for Class I-V restorations (BEAUTIFIL Flow Plus, Shofu Dental Corporation; G-aenial Universal Flo, GC America; GrandioSO Heavy Flow, VOCO America), as well as for provisional constructions (4), (5), (6). The high filler content increases strength and wear resistance, lowers polymerization shrinkage, and allows the material to be stacked. The addition of nanofill particles assists in creating highly esthetic restorations (7). The use of flowable composite as the first increment at the bottom of the proximal box of a Class II preparation which is one of the most popular contemporary uses of flowable composites, was not even mentioned as an indication for Revolution and sometimes flowable composites are even used on the top of esthetic restoration in order to improve occlusal morphology. Although the newest generations of flowables overcome the disadvantages of the preceding ones, there is a reasonable concern that flowable composites may not be wear resistant enough to restore contact areas.

The experiments of the present study were carried out using the "Sofia" in vitro chewing simulator (Fig. 1), which has been described in several previous articles $(8,9)$. Its construction principles are based on the Functional Occlusion Concept developed by Le Gall and Lauret in 1996 (10). The most significant aspect in which the functional occlusion concept differs from classical occlusodontics, is the direction of the chewing cycle and the presence of additional occlusal contacts which occur during physiological chewing and are different from the ones that can be registered in centric occlusion and excursive movements. Since the physiological chewing cycle (during the natural act of chewing) has a centripetal direction of movement, we chose the model of dynamic teeth contact and dynamic load, as proposed by Le Gall and Lauret, to be followed for the purpose of the in-vitro tests (Fig. 2). 


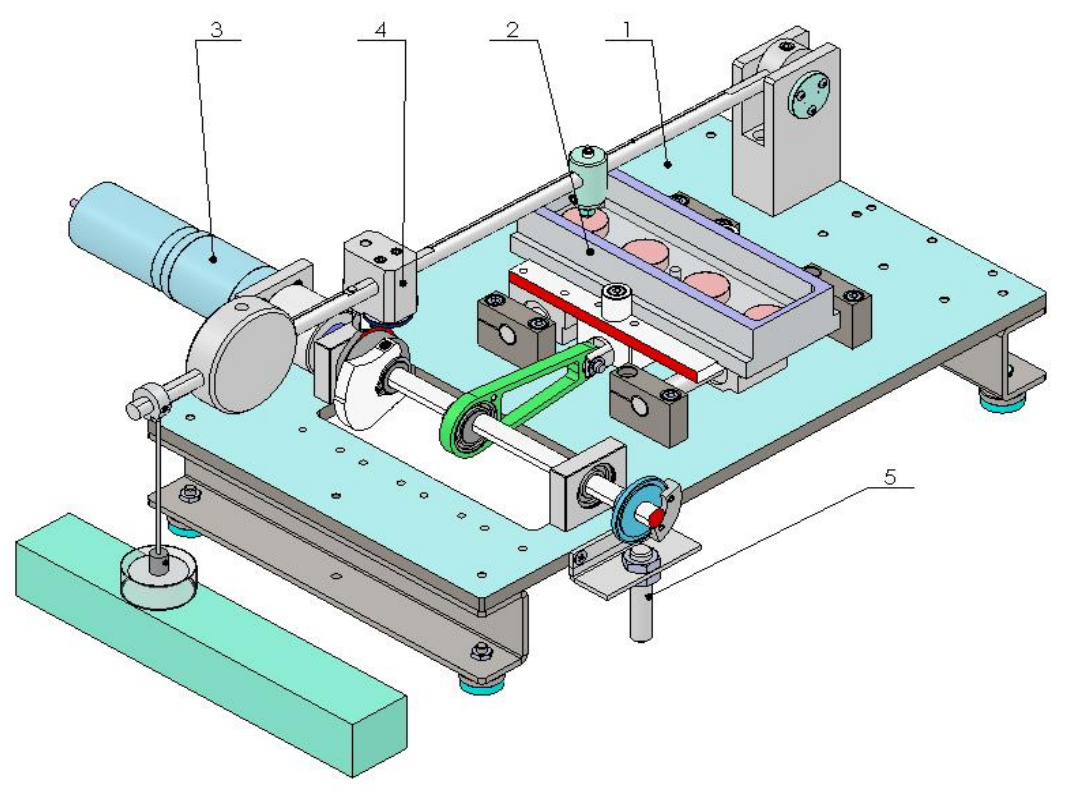

Fig. 1 - Simplified scheme of the "Sofia" chewing simulator: base (1), a carriage (2) with a fixed tray housing 4 standardized flat samples of the tested material in an environment that might be distilled water (for twobody wear tests) or an artificial food medium (for three-body wear tests), a rotating shaft (3) on which a magnetic cam and an alleviating cam are mounted, and a rocker arm (4) transmitting the motion of the rotating shaft to the antagonist.
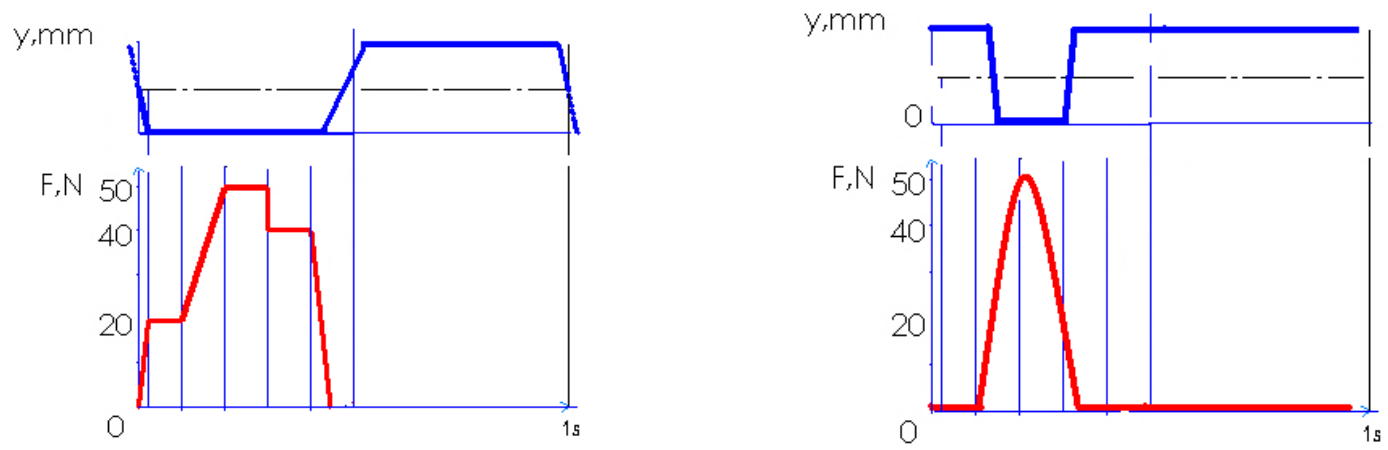

Fig. 2 - The cyclogram represents one experimental chewing cycle reproduced by the chewing simulator. The horizontal line (the $x$-axis) of the two diagrams represents the time of one experimental cycle. The diagram in red shows the change of force over time, while the blue one measures the distance between the antagonist and the tested specimen. When the blue line coincides with the x-axis on the upper diagram the antagonist in is contact with the specimen. Only during this interval the loading force changes. In the second 
part of the experimental cycle the antagonist and the specimen are separated - this corresponds to the part of the chewing cycle when the teeth are out of contact. The cyclogram to the left represents an experimental cycle carried out according to the functional occlusion concept $(A)$; the cyclogram to the right represents an experimental cycle based on the loading profile according to the classical gnathological concept (B). The "Sofia" chewing simulator is capable of reproducing both loading profiles, but for the purpose of this study we chose to use the first one.

The dynamic load curve is achieved by using the interaction between a permanent magnet and a lasershaped ferromagnetic cam, which reproduce the dynamic load curve typical of the physiological chewing process. A second rotating cam determines the periodic ascent and descent of the antagonist towards the tested material to mimic the repetition of contact and non-contact phase of the chewing cycles.

\section{Aim}

The purpose of this study was to compare wear resistance for flowable and heavily filled composites and therefore to demarcate the indication for use of flowable composites.

\section{Materials and methods}

Two specimens were fabricated from flowable composite (SDI Wave A3, LOT 131230N 0297 and SDI Wave A2 LOT 131081N 0297) and two specimens from heavily filled composite (Herculite HRV, LOT5592354, Kerr Italia Srl.). For this purpose metal rings with an outer diameter of $25 \mathrm{~mm}$, inner diameter $24 \mathrm{~mm}$ and 8 $\mathrm{mm}$ height were used. The metal rings were placed in a specially designed holder, which was tightly fixed onto a glass slab with a smooth surface. 2-2.5 grams of the tested material was placed into the ring and carefully adapted against the glass slab until a uniform layer of about 1-2 mm was achieved. The polymerization of the material was done with a $2000 \mathrm{~mW} / \mathrm{cm} 2$ light-curing lamp. The surface of the samples was divided in 9 areas (with a size roughly coinciding to that of the light-transmitting tip of the lamp.) The polymerization was done separately for each area using two polymerization modes - first with the "soft start" mode for 20 seconds (with low light intensity at the beginning and a gradual increase of intensity towards the end of the curing cycle) and then with the pulse mode (with light impulses at a maximum intensity followed by a period of lack of light irradiation). Subsequently the remaining volume of the sample was filled up with pink cold-curing resin (Duracryl, Spofa Dental).

All specimens were subjected to tests in distilled water.

Prefabricated silicon nitride spheres with a diameter of $4 \mathrm{~mm}$ were used as antagonist for the tests. The spheres were mounted on a special holder. The measurement was performed using a contact profilometer (with a vertical accuracy of $1 \mu \mathrm{m}$ and horizontal accuracy of $5 \mu \mathrm{m}$ ), following a predetermined mesh. Each wear groove on the specimens' surface was divided in 10 sections. Each section was measured in 3 points - 30 points per groove in total.

In order to diminish the role of errors during the sample fabrication and fixation the depth of wear produced throughout the experiment was calculated using the following formula:

$$
Z=Z M-(Z L+Z D) / 2
$$


where $Z$ is the depth of the wear groove; $Z A$ - is the referent plane (where the initial point $A$ is situated); $Z M$ - the distance from the deepest point to the referent plane; ZL and ZD are accordingly the distances measured from the left and right bord of the groove to the referent plane (these were measured to equal distances to the left and right).

The data was recorded and processed with the statistical package IBM SPSS Statistics 22.0.

$P<0,05$ was set as the significance level at which the zero hypothesis would be rejected. The following methods were applied:

1. Descriptive analysis

2. Analysis of variance

3. Nonparametric Kolmogorov-Smirnov test and Shapiro-Wilk test

4. Nonparametric Kruskal-Wallis test

5. Nonparametric Mann-Whitney test

6. Independent Samples T-Test

7. One-way ANOVA

\section{Results}

The results are presented in table 1. Following conclusions can be drawn:

- Herculite HRV (KERR) is more wear resistant than WAVE SDI as the average depth of wear grooves of the former is $53 \mu$ and of the latter $-58 \mu$.

- There is a significant difference between the wear resistance of the two tested materials $(p<0,1)$.

Table 1: Comparison between the depth of wear grooves in conventional and flowable composite samples

\begin{tabular}{lcccccc}
\hline Material & Sample & Y & $\begin{array}{c}\text { Number of } \\
\text { experiments }\end{array}$ & $\bar{X}$ & SD & V \\
\hline Herculite HRV & $1-2$ & $1-6$ & 72 & $52,66^{\mathrm{a}}$ & 39,69 & 75,37 \\
WAVE SDI & $3-4$ & $1-6$ & 72 & $58,02^{\mathrm{a}}$ & 32,89 & 56,69 \\
\hline
\end{tabular}

- $\quad$ the identical letters mean absence of significant difference $(p=0.093)$. 


\section{Discussion}

The wear rate of dental materials concerns every clinician since this factor is tightly connected to restorations' longevity and may be a potential predictor of their deterioration. It determines the indication for use of restorative materials either for posterior or anterior regions, for small or large cavities, for high or low stress areas and etc. In the present study the wear rate of one flowable (WAVE SDI) and one conventional (Herculite HRV) composites are compared in order to gain better insight into their application limitations. Some manufacturers suggest flowable composites for use as liners in areas of difficult access such as irregular internal surface and proximal boxes of Class II preparations. The assumptions are that these less viscous materials flow easily into, adapt to and fill the tooth surface, resulting in less leakage and postoperative sensitivity $(11,12,13)$. Flowables help reduce microleakage at cervical margins (15). While flowable composites may provide better adaptation, they may also act as a flexible intermediate layer, which helps relieve stresses during polymerization shrinkage of the restorative resin $(15,16)$. However, flowable composites are reported to shrink more than traditional composites because they have less filler loading (17). Perhaps the relatively thin layer minimizes this effect. As this research confirms, the wear rate of flowable composites was higher than that of packable composites; therefore, flowable composites should be used only at contact-free areas $(18,19)$. Current flowable materials can easily be syringed into the cavity but are sometimes difficult to manipulate because of their stickiness. Air is sometimes trapped in the restorations while removing the syringe tip from the cavity. Porosities were also observed in packable composite restorations so the operators need to use these materials with caution.

\section{Conclusion}

The present study reveals that flowable composites are less wear resistant than conventional composites. Therefore it is advisable to use flowables at low-stress and contact-free areas, as a protective adhesive layer in Class II restoration (open sandwich technique), by fissurotomy or small Class I decays in Pediatric dentistry. Their application on the occlusal surface as an morphology refinement is rather unfavorable.

\section{References}

1. Nancy L. Clelland, Matthew P. Pagnotto, Ronald E. Kerby, Robert R. Seghi Relative wear of flowable and highly filled composite The Journal of Prosthetic Dentistry February 2005Volume 93, Issue 2, Pages 153-157Lila AR, Sarathi V, Jagtap V, et al. Renal manifestations of primary hyperparathyroidism. Indian J Endocrinol Metab. 2012 Mar-Apr; 16(2): 258-262.

2. https://www.dentaledglobal.com/media/PRODUCT_FILES/2015/02/07/Flowable_Composites.pdf

3. Qamar Z, Fatima T. Comparative Review Of Various Flowable Composites. J Pak Dent Assoc 2014; 23(1):11-14

4. Preliminary constructions in fixed prosthetic dentistry, Dissertation for acquiring educational and scientific degree "Doctor", Sofia, 2003

5. Dimova, M., I. Borisov, V. Atanasov: An in-vitro investigation of exothermic reactions in polymerization of seven materials used for provisional prosthetic constructions, Problems of Dental Medicine, Sofia, XXXIV/2008 - part II, 63-70

6. Dimova, M.: An in-vivo investigation of exothermic reactions in polymerization of four materials used for provisional prosthetic constructions - "Tempofit@" /Detax/, "Structur 2 SC/QM" /Voco/, 
"Luxatemp ${ }^{\circledR}$ - Automix plus" /DMG/, "Jet Tooth Shade" /Lang Dental/. Pharmacologyonline, 2011, $3,524-535$

7. http://www.firstimpressionsmag.com/dental-advisor-resin-composites.html

8. Chakalov I, Ivanova P, Apostolov N. Influence of different tribological mechanisms on in- vitro wear simulation results of composites. MedInform 2014, vol 1, Issue 1,28-34

9. Chakalov I, Ivanova P. In vitro wear resistance of five composite restorative materials: A comparative research study. Journal of the Lebanese Dental Association. Volume 52- № 1 January-June 2017; p47-53.

10. Lauret J-F, Le Gall MG. The function of mastication - A key determinant of Dental Occlusion. Practical Periodontics and Aesthetic Dentistry. September/October; Vol 8. No8. 1996

11. Bayne SC, Thompson JY, Swift EJ, Stamatiades P\& Wilkerson M (1998) A characterization of firstgeneration flowable composites Journal of the American Dental Association 129(5) 567-577

12. Leinfelder KF \& Prasad A (1998) A new condensable composite for the restoration of posterior teeth Dentistry Today 17(2) 112-116

13. The dental advisor (1999) Packable composites The Dental Advisor 16(9) 1-4

14. Leevailoj C, Cochran MA, Matis BA, Moore BK, Platt JA. Microleakage of Posterior Packable Resin Composites With and Without Flowable Liners.Operative Dentistry, 2001, 26, 302-307

15. Kemp-Scholte CM \& Davidson CL (1990) Complete marginal seal of Class V resin composite restorations effected by increased flexibility Journal of Dental Research 69(6) 1240- 1243

16. Rooklidge E, Boyer D\& Bouschlicher M (1999) Cusp deformation by shrinkage of condensable composite Journal of Dental Research 78 Abstracts of Papers p 399 Abstract 2349

17. Tolidis K \& Setcos JC (1999) Initial degree of polymerization shrinkage exhibited by flowable composite resins Journal of Dental Research 78 Abstracts of Papers $p 482$ Abstract 3015

18. Bayne SC, Thompson JY, Swift EJ, Stamatiades P\& Wilkerson M (1998) A characterization of firstgeneration flowable composites Journal of the American Dental Association 129(5) 567-577

19. Dang HM \& Sarrett DC (1999) Wear behavior of flowable and condensable composite resins Journal of Dental Research 78 Abstracts of Papers p 447 Abstract 2736

\section{Corresponding author:}

Assistant Professor Ivan Chakalov,

Prosthetic Dentistry,

Faculty of Dental Medicine,

Medical University - Sofia

1 G. Sofiiski Str., 1431

Sofia, Bulgaria

email: uanio@yahoo.com 